\title{
COVERING HOMOTOPY PROPERTIES OF MAPS BETWEEN C.W. COMPLEXES OR ANR'S
}

\author{
MARK STEINBERGER AND JAMES WEST
}

\begin{abstract}
Serre fibrations between C.W. complexes are fibrations in the category of compactly generated weak Hausdorff spaces, but not, by example, in the category of all spaces. Under suitable hypotheses the notions of Dold fibration in the two categories coincide. Examples are given of maps with ANR base, total space and fibres which are not Dold fibrations but whose pullbacks over maps out of locally finite-dimensional paracompact spaces are bundles.
\end{abstract}

1. Introduction. We were motivated here by our work on classifying spaces of monoids of self-maps of Hilbert cube manifolds.

In [5], R. Brown gives two Serre fibrations that are not Hurewicz fibrations, remarking that he cannot find any in the literature. He also asks whether a Serre fibration whose fibres are C.W. complexes or ANR's is a Hurewicz or Dold (weak) fibration.

Allaud [1] gives counterexamples with one-point fibres. However, the base space is either not compactly generated or not locally connected.

Let $p: E \rightarrow B$ be a map. We say that $p$ is an LFD-fibration if the pullback of $p$ over any map $f: X \rightarrow B$, with $X$ a locally finite-dimensional paracompact space, is a Hurewicz fibration. Let $\mathcal{Q}$ be the category of compactly generated weak Hausdorff spaces. A $\mathscr{U}$-fibration is a map in $\mathscr{U}$ which has the covering homotopy property for spaces in $\mathcal{Q}$. (Many homotopy theorists call such maps Hurewicz fibrations.) We give the following counterexamples to Brown's question.

EXAMPLE 1. We give a simplicial map of finite-dimensional simplicial complexes which is a 2 -fibration but not a Hurewicz fibration.

EXAMPLES 2 AND 3. We give LFD fibrations with compact Hilbert cube manifold base which are not Dold fibrations. The total space and fibres are Hilbert manifolds in Example 2 and locally compact Hilbert cube manifolds in Example 3, hence separable, metric ANR's.

Examples 2 and 3 are more or less in the folklore of infinite-dimensional topology. As expected, their finite-dimensional analogues have no such anomalies, and they help motivate the following questions.

Received by the editors January $31,1983$.

1980 Mathematics Subject Classification. Primary 55R05, 54E60; Secondary 57N20, 55M15.

Key words and phrases. Serre fibration, Dold fibration, Hurewicz fibration, covering homotopy property, C.W. complex, Hilbert cube manifold, Kan fibration, geometric realization, singular complex, compactly generated. 
Question 1. Is a Serre fibration over a finite complex with (compact) ANR total space and fibres a Hurewicz fibration?

Question 2. Is an LFD-fibration with compact Hilbert cube manifold base and compact ANR total space and fibres a Hurewicz fibration?

We have the following positive results.

THEOREM 1. A Serre fibration between C.W. complexes is a regular थ-fibration.

Note that we require no compatibility between the cell structures of the total space and base and that the fibres need not be C.W.

Under the right hypotheses, a $\mathcal{Q}$-fibration is a Dold fibration.

THEOREM 2. Let $p: E \rightarrow B$ be a map in $थ$ which satisfies the weak covering homotopy property for spaces in $\mathcal{Q}$. If the base and either the fibres or the total space have the homotopy type of a C.W. complex, then $p$ is a Dold fibration.

Quillen in [17] shows that the geometric realization of a Kan fibration is a Serre fibration. Theorem 1 now gives us the following, which would also follow from Quillen's proof.

COROLlARY 1. The geometric realization of a Kan fibration is a regular $\mathcal{Q}$-fibration.

Corollary 2. A map $p: E \rightarrow B$ is a Serre fibration if and only if the induced map of geometric realizations of total singular complexes is a regular $\mathcal{Q}$-fibration.

In fact, Theorem 1 is applicable to more general situations, even when restricted to maps of simplicial sets, as proper maps are rarely Kan fibrations (e.g., the projection of $B \times S^{1}$ onto $B$ for any $B$ ).

The restriction to $\mathcal{Q}$ in Corollary 2 is necessary.

Proposition 1. Let $p: E \rightarrow B$ be a Serre fibration such that $B$ contains a nontrivial path $\alpha$ and that the fibre over $\alpha(0)$ contains a nontrivial path $\beta$. Then the induced map of geometric realizations of total singular complexes is not a Hurewicz fibration.

2. The examples.

EXAMPLE 1. Let $K$ and $L$ be Dowker's examples [9] of two simplicial complexes on whose product the Tikhonov topology is strictly coarser than the C.W. topology, and let $E$ denote $K \times L$ with the C.W. topology. Then the projections $p: E \rightarrow K$ and $\bar{p}$ : $E \rightarrow L$ are Serre fibrations between simplicial complexes that are not Hurewicz fibrations.

Proof. We prove the case of $p: E \rightarrow K$. The other is identical. Let $N$ denote the positive integers. Then $K$ is the cone on the discrete set $N^{N}$ with vertex $u$ and $L$ is the cone on $N$ with vertex $v$. We parametrize an edge by $[0,1]$ with the cone point corresponding to 0 and write $t x$ for the point on the edge between the cone point and $x$ corresponding to $t \in[0,1]$. Let $X=K \times L$ with the Tikhonov topology and let $A=K \times v \cup u \times L$ be the wedge. Then $A$ is a strong deformation retract of $X$, so the inclusion $A \subset X$ is a cofibration. If $p$ were a Hurewicz fibration, then the map $g: X \times 0 \cup A \times I \rightarrow E$ defined by $g(k, 1, t)=(k, t 1)$ would extend to a map $G$ : $X \times I \rightarrow E$ covering the projection $\pi: X \times I \rightarrow K$. The restriction $g_{1}$ of $G$ to $X \times 1$ would be a fibrewise map of $X$ to $E$ which extends the inclusion $A \subset E$. 
No such function is continuous as follows. Dowker [9] shows that any neighborhood of $(u, v)$ in $X$ contains a product $[u, x / x(y)] \times[v, y / x(y)]$ for some $x \in N^{N}$ and $y \in N$. Since $g_{1}$ is the identity on $u \times L$ and covers the projection onto $K$, one can choose, for each $x$ and $y$, an $\varepsilon_{x, y} \in(0,1 / x(y))$ which is strictly less than the $L$-coordinate of $g_{1}\left(\varepsilon_{x, y} x, y / x(y)\right)$. Thus, if $W$ is the neighborhood of $(u, v)$ in $E$ whose intersection with $[u, x] \times[v, y]$ is bounded by the linear paths from $\left(\varepsilon_{x, y} x, \varepsilon_{x, y} y\right)$ to $(x, v)$ and $(u, y)$, then $g_{1}^{-1}(W)$ is not open in $X$.

REMARK. The fibres of $p$ are countable finite-dimensional simplicial complexes, as is the base of $\bar{p}$.

EXAMPLE 2. Let $M$ be a compact, connected Hilbert cube manifold and let $H(M)$ be the space of self-homeomorphisms of $M$ with the compact-open topology. Let $x \in M$ and let $\varepsilon: H(M) \rightarrow M$ be evaluation at $x(\varepsilon(h)=h(x))$. Then

(i) $\varepsilon$ is an LFD-fibration.

(ii) The total space and fibres are Hilbert manifolds. The fibres are collared in the total space.

(iii) $\varepsilon$ has no local sections, hence is not a Dold fibration.

Proof. (i) This follows from Michael's Selection Theory [16], since the customary proofs $[3,7]$ of the homogeneity of the Hilbert cube and the local contractibility of $\varepsilon^{-1}(x)[13]$ are strong enough to show that $\left\{\varepsilon^{-1}(m)\right\}_{m \in M}$ is equi- $L C^{n}$ for each $n$ in Michael's terminology [16]. Thus, the pullback of $E$ over $f: X \rightarrow M$ admits local sections and is a principal $\varepsilon^{-1}(x)$-bundle.

(ii) That $H(M)$ and $\varepsilon^{-1}(x)$ are Hilbert manifolds (and absolute neighborhood extensors for metric spaces) is shown in [14 and 19]. That $\varepsilon^{-1}(x)$ is collared in $H(M)$ follows from [3, XIII, $\S 4 ; 2$ and 4], once we prove that each map $\alpha: I^{k} \rightarrow H(M)$ can be approximated arbitrarily closely by maps missing $\varepsilon^{-1}(x)$ for $k \geqslant 0$. Consider $\varepsilon \circ \alpha: I^{k} \rightarrow M$. Since $M$ is a Hilbert cube manifold, $x$ is a $Z$-set in $M$ and we may choose an arbitrarily small homotopy from $\varepsilon \circ \alpha$ to a map which misses $x$. By (i), the pullback of $\varepsilon$ over this homotopy is a trivial bundle, and the result follows. For $m \neq x$, multiplication on the left by $g \in \varepsilon^{-1}(m)$ induces an ambient homeomorphism from $\varepsilon^{-1}(x)$ to $\varepsilon^{-1}(m)$, so these results carry over to $\varepsilon^{-1}(m)$.

(iii) Chapman [6] proved this for the special case $M=Q$, the Hilbert cube. We reduce it via [13] as follows. As above, it suffices to show $\varepsilon$ admits no section in a neighborhood of $x$. Suppose $\sigma: U \rightarrow H(M)$ is a section, with $U$ a neighborhood of $x$. We may assume $\bar{U}$ is the Hilbert cube and $\sigma(x)=1$. Let $V$ be a neighborhood of the boundary of $U$ which misses an entire neighborhood $W$ of $x$. By [12], there is a deformation of a neighborhood of 1 in $H(M), \Phi: N \times I \rightarrow H(M)$, into the subspace of $H(M)$ which fixes the boundary of $U$, which doesn't alter the maps outside $V$. Then $\sigma_{1}(m)=\Phi(\sigma(m, 1))$ induces a local section of $\varepsilon: H(\bar{U}) \rightarrow \bar{U}$ on $W \cap \sigma^{-1}(N)$.

REMARK. For finite-dimensional manifolds $M$, the analogous map $\varepsilon: H(M) \rightarrow M$ is a principal $\varepsilon^{-1}(x)$-bundle [12].

EXAMPLE 3. Let $p_{1}: Q \times Q \rightarrow Q$ be the projection onto the first factor, where $Q$ is the Hilbert cube and let $\Delta \subset Q \times Q$ be the diagonal. Let $E=Q \times Q-\Delta$ and let $p$ : $E \rightarrow Q$ be induced by $p_{1}$. Then

(i) $p$ is an LFD-fibration.

(ii) The fibres of $p$ are homeomorphic to $Q \times[0,1)$ and are collared in $E$. 
(iii) $p$ admits no global section, hence is not a Dold fibration.

Proof. (i) Let $f: X \rightarrow Q$ with $X$ locally finite dimensional and paracompact and let $x \in X$. Let $\varepsilon: H(Q) \rightarrow Q$ be evaluation at $f(x)$. A local section of $f^{*}(\varepsilon)$ gives a local trivialization of $f^{*}(p)$.

(ii) This follows from [7, Theorems 11.1, 12.2, 16.2 and 3.1].

(iii) If $p_{2}: E \rightarrow Q$ is induced by projection onto the second factor and $\sigma: Q \rightarrow E$ is a section of $p$, then $p_{2} \circ \sigma$ has no fixed points, contradicting the fixed point property of compact AR's. (This is also the main ingredient of Example 2(iii).)

REMARK. (i) While $\Delta \subset Q \times Q$ is a $Z$-set, it is not a fibred $Z$-set over $p_{1}$ in the sense of [14], as then $\bar{p}$ would be a bundle map.

(ii) The finite-dimensional analogue $p: I^{k} \times I^{k}-\Delta \rightarrow I^{k}$ is not even a quasifibration, as the fibres have different homotopy types, but the restriction over the interior of $I^{k}$ is a bundle by the remark after Example 2.

\section{Proofs of Theorems 1 and 2 and Proposition 1.}

Proof of Theorem 1. Let $p: E \rightarrow B$ be a Serre fibration between C.W. complexes. We show that if we take products in $\mathscr{Q}$, then $p$ is locally trivial in the sense of [10, Chapter XX, §4]. Thus, if the path space on $B$ is also given the compactly generated topology, there is a regular lifting function for $p$.

Let $U$ be a contractible open set in $B$. Since $U$ may be given the structure of a C.W. complex it is equiconnected in $\mathcal{Q}[11]$, i.e. there is a homotopy

$$
H: U \times U \times I \rightarrow U
$$

between the two projection maps which is constant on the diagonal. If $K: p^{-1} U \times U$ $\times I \rightarrow p^{-1} U$ is a regular lift of $H(p \times 1 \times 1)$, then the restriction of $K$ to $p^{-1} U \times U \times 1$ is a slicing function over $U$. Since $p^{-1} U \times U$ is a C.W. complex and the space of constant paths in $B$ is a zero set in the space of all paths (e.g., take the diameter of a path in a continuous metric induced by an injection from $B$ into a Hilbert space whose basis has the same cardinality as the set of cells of $B$ ), a regular lift exists (cf. [20]).

Proof of Theorem 2. Of course, the arguments of [8 and 18] may be restricted to $\mathscr{Q}$, so that $p$ is locally fibre homotopy equivalent in $\mathcal{Q}$ to a product $B \times L$, with $L$ a simplicial complex. It suffices to show the two topologies on the product are fibre homotopy equivalent. Let $f: L \rightarrow L$ be the simplicial map from the second derived to the first derived which takes a vertex $\sigma_{0}<\cdots<\sigma_{k}$ to the barycenter of $\sigma_{0}$. It suffices to show $1 \times f$ is continuous from the Tikhonov topology to the compactly generated topology. Let $W$ be a neighborhood of $(x, f(y))$ in the latter topology and let $\sigma$ be the carrier of $y$. Since the two topologies agree on $B \times \sigma$, there is a product neighborhood $U \times V$ of $(x, f(y))$ in $W \cap B \times \sigma$. Then $U \times f^{-1} V$ is a neighborhood of $(x, y)$ in $(1 \times f)^{-1}(W)$.

Proof of Proposition 1. Again, products are given the C.W. topology. Let $\Delta^{\infty}$ be the infinite simplex and let $K$ be the space of Example 1. Let $X$ be the space obtained from $K \times \Delta^{\infty}$ by identifying $N^{N} \times \Delta^{\infty}$ to $N^{N}$ under the projection map and let $q: X \rightarrow K$ be induced by the projection. Since $q$ is a fibrewise retract of the projection, it is the geometric realization of a Kan fibration $q_{*}: X_{*} \rightarrow K_{*}$. If one applies the analogous construction to Example 1, the induced map is not a Hurewicz 
fibration, by the proof of Example 1. This map is a fibrewise retract of $q$, hence $q$ is not Hurewicz. Since $q_{*}$ is $\mathrm{Kan}$ and $X_{*}$ is simplicially contractible, $q_{*}$ is a fibrewise retract of any simplicial map over $K_{*}$ which extends it, so it suffices to embed $q_{*}$ in the total singular complex of $p$. Let $j: \Delta^{\infty} \rightarrow I$ be a map whose restriction to each finite face is nondegenerate and apply the Serre property to $\beta \circ j$ and any inclusion of $K$ in the total singular complex of $\alpha$.

\section{REFERENCES}

1. G. Allaud, On an example of R. Brown, Arch. Math. 19 (1968), 654-655.

2. R. D. Anderson and J. McCharen, On extending homeomorphisms to Fréchet manifolds, Proc. Amer. Math. Soc. 25 (1970), 283-289.

3. Cz. Bessaga and A. Pelczynski, Selected topics in infinite dimensional topology, Monografie Mat., Vol. 58, PWN, Warsaw, 1975.

4. M. Brown, Locally flat embeddings of topological manifolds, Topology of 3-Manifolds and Related Topics, Prentice-Hall, Englewood Cliffs, N. J., 1962, pp. 83-91.

5. R. Brown, Two examples in homotopy theory, Math. Proc. Cambridge Philos. Soc. 62 (1966), $575-576$.

6. T. A. Chapman, Canonical extensions of homeomorphisms, Topology Appl. 2 (1972), 227-247.

7. __ Lectures on Hilbert cube manifolds, CBMS Regional Conf. Ser. in Math., no. 28, Amer. Math. Soc., Providence, R. I., 1976.

8. A. Dold, Partitions of unity in the theory of fibrations, Ann. of Math. (2) 78 (1963), 223-255.

9. C. H. Dowker, Topology of metric complexes, Amer. J. Math. 74 (1952), 555-577.

10. J. Dugundji, Topology, Allyn and Bacon, Boston, Mass., 1966.

11. E. Dyer and S. Eilenberg, An adjunction theorem for locally equiconnected spaces, Pacific J. Math. 41 (1972), 669-685.

12. R. D. Edwards and R. C. Kirby, Deformations of spaces of embeddings, Ann. of Math. (2) 93 (1971), 63-88.

13. A. Fathi and Y.-M. Visetti, Deformation of open embeddings of $Q$-manifolds, Trans. Amer. Math. Soc. 224 (1976), 427-436.

14. S. Ferry, The homeomorphism group of a compact Hilbert cube manifold is an ANR, Ann. of Math. (2) 106 (1977), 101-119.

15. R. Luke and W. K. Mason, The space of homeomorphisms on a compact 2-manifold is an absolute neighborhood retract, Trans. Amer. Math. Soc. 164 (1972), 275-285.

16. E. Michael, Continuous selections. II, Ann. of Math. (2) 64 (1956), 562-580.

17. D. Quillen, The geometric realization of a Kan fibration is a Serre fibration, Proc. Amer. Math. Soc. 19 (1968), 1499-1500.

18. J. D. Stasheff, A classification theorem for fibre spaces, Topology 2 (1963), 239-246.

19. H. Torunczyk, Homeomorphism groups of Hilbert cube manifolds which are manifolds, Bull. Acad. Polon. Sci. Sér. Sci. Math. Astronom. Phys. 25 (1977), 401-408.

20. P. Tulley, On regularity in Hurewicz fiber spaces, Trans. Amer. Math. Soc. 116 (1965), 126-134.

Department of Mathematics, Cornell University, Ithaca, New York 14853

Current address (Mark Steinberger): Department of Mathematical Science, Northern Illinois University, De Kalb, Illinois 60115

Current address (James West): Institute for Advanced Study, Princeton, New Jersey 08540 